\title{
Thio-click approach to the synthesis of stable glycomimetics ${ }^{\ddagger}$
}

\author{
${ }^{a}$ László Lázár, ${ }^{\mathrm{b}}$ Magdolna Csávás, ${ }^{\mathrm{c}}$ Marietta Tóth, ${ }^{\mathrm{c}}$ László Somsák, \\ b Anikó Borbás* \\ a Department of Applied Chemistry, University of Debrecen, Egyetem tér 1, H-4032 Debrecen, Hungary \\ ${ }^{\mathrm{b}}$ Department of Pharmaceutical Chemistry, University of Debrecen, PO Box 70, H-4010 Debrecen, Hungary \\ ${ }^{\mathrm{c}}$ Department of Organic Chemistry, University of Debrecen, PO Box 20, H-4010 Debrecen, Hungary
}

Received 25 September 2014; Revised 18 November 2014; Accepted 24 November 2014

\begin{abstract}
Carbon-sulfur-bridged glycomimetics were prepared by free radical hydrothiolation of the exocyclic double bond of unsaturated sugars. Reaction between benzoyl-substituted pyranoid-exoglycal and a range of thiols including peptide, 1-thioglycerol and 1-thiosugar derivatives gave $\beta$-Dconfigured carbon-sulfur-linked glycoconjugates with full stereoselectivity. Addition of a panel of thiols to a 3-exomethylene-glucofuranose derivative also proceeded in a stereoselective manner and afforded a series of D-allo-configured 3-deoxy-3-C-S-bridged glycoconjugates.
\end{abstract}

(C) 2015 Institute of Chemistry, Slovak Academy of Sciences

Keywords: exoglycal, 3-exomethylene-furanose hydrothiolation, photocatalysis, radical, glycopeptide, glycoconjugate

\section{Introduction}

Carbohydrates play an important role in diverse biological processes, including inflammation, immune response, cancer metastasis, as well as viral and bacterial infection and, in principle, offer immense opportunities for therapeutic drug development. However, the instability of glycosides toward chemical and enzymatic degradation due to the readily hydrolysable native glycosidic bond hampers the in vivo applications of carbohydrate-based molecules. Therefore, there has been a long-standing interest in the synthesis of hydrolytically stable carbohydrate mimetics that can be used as leads for new therapeutic agents and probes in biological studies (Ernst \& Magnani, 2009).

Thiosugars containing a sulfur atom instead of an oxygen atom in the ring are stable monosaccharide mimics and have gained importance in glycobiology and as potential drugs (Robina et al., 2001; Witzack \& Culhane, 2005). The best-documented stable gly- comimetics are the $C$ - and $S$-glycosides, in which the glycosidic oxygen is replaced by a methylene group or a sulfur atom (Liu et al., 2001; Szilágyi \& Varela, 2006; Witczak et al., 2007). Research efforts have also been directed toward the incorporation of a novel, stable linker comprising two or three bridging atoms in place of a native $O$-glycosidic bond. Hence, among others, disulfide- (Szilágyi et al., 2001; Illyés et al., 2011), seleno-sulfide-, (Chakka et al., 2005), sulfonamide(Lopez et al., 2011) or urea-bridged glycosides (Prosperi et al., 2004) have been prepared as novel carbohydrate derivatives with potential bioactive properties.

Over the last few years, photoinduced free-radical addition of thiols to alkenes, termed thiol-ene coupling or thiol-ene click reactions, has emerged in the field of carbohydrate chemistry as a robust ligation tool providing an easy access to $S$-linked glycoconjugates (Dondoni \& Marra, 2012; Witczak \& Bielski, 2013). Interestingly, there are very few examples of the incorporation of unsaturated carbohydrates bearing an

*Corresponding author, e-mail: borbas.aniko@pharm.unideb.hu

$\ddagger$ Presented at the 3th Bratislava Symposium on Saccharides "Recent Advances in Glycomics", Smolenice Castle, Slovakia, 22-26 June, 2014. 
exo- or endocyclic double bond within the thiol-ene coupling strategy (Fiore et al., 2009; Lázár et al., 2012, 2013; Staderini et al., 2012), and the inherent potential of this mild and efficient synthetic methodology of a sugar unit incorporation into another bioactive compound through a carbon-sulfur linker has remained unexploited until now.

Here, we demonstrate the benefits of free-radical hydrothiolation of alkenyl sugars bearing an exocyclic double bond providing stable carbon-sulfur-bridged glycomimetics.

\section{Experimental}

Optical rotation was measured at ambient temperature with a Perkin-Elmer 241 automatic polarimeter. TLC was performed on a Kieselgel $60 \mathrm{~F}_{254}$ (Merck, USA) with detection using a 5 vol. \% ethanolic sulfuric acid solution and heating. Column chromatography was performed on Silica gel 60 (Merck, Germany, 0.063-0.200 mm). Organic solutions were dried over $\mathrm{MgSO}_{4}$, and concentrated in vacuum. The ${ }^{1} \mathrm{H}$ NMR $(360 \mathrm{MHz}$ and $400 \mathrm{MHz})$ and ${ }^{13} \mathrm{C} \mathrm{NMR}(90.54 \mathrm{MHz}$ and $100.28 \mathrm{MHz}$ ) spectra were recorded with a Bruker Avance DRX-360 and a DRX-400 spectrometer, respectively, at $25^{\circ} \mathrm{C}$ (Germany). Chemical shifts are referenced to $\mathrm{Me}_{4} \mathrm{Si}$ or DSS as parts per million (0.00 ppm for $\left.{ }^{1} \mathrm{H}\right)$ and to the solvent signals $\left(\mathrm{CDCl}_{3}: 77.00\right.$ ppm for $\left.{ }^{13} \mathrm{C}\right)$. The ${ }^{1} \mathrm{H}$ and ${ }^{13} \mathrm{C}$ NMR assignments were established from 1D NMR spectra. Elemental analyses (C, H, S) were performed using an Elementar Vario MicroCube instrument (Germany).

\section{General method for photoinduced addition of thiols (IIIa-IIIh) to glycals I and II}

To a solution of the starting unsaturated monosaccharide $(1.00 \mathrm{mmol})$ in the specified solvent $(7 \mathrm{~mL})$, thiol (2.0 eq.) and 2,2-dimethoxy-2-phenylacetophenone (DPAP, $25 \mathrm{mg}, 0.10 \mathrm{mmol}$ ) were added. The solution was irradiated at ambient temperature for $15 \mathrm{~min}$. Then, it was concentrated and the residue was purified using column chromatography.

Using IIIf as the thiol, an $1: 2$ thiol/alkene ratio was applied and the DPAP addition and irradiation were repeated twice more.

\section{Compound IVa}

Compound $I(100 \mathrm{mg}, 0.169 \mathrm{mmol})$ and $N$-acetylcysteine IIIa $(55 \mathrm{mg}, 0.338 \mathrm{mmol})$ were reacted in toluene-MeOH 1 : 1 vol. (6 mL) according to the general method. The crude product was purified by silica gel chromatography in $\mathrm{CH}_{2} \mathrm{Cl}_{2}-\mathrm{MeOH}\left(\varphi_{\mathrm{r}}=\right.$ $85: 15), R_{\mathrm{f}}=0.38$, to give $I V a(113 \mathrm{mg}, 88 \%)$ as a colorless syrup. $[\alpha]_{\mathrm{D}}\left(c=0.16, \mathrm{CHCl}_{3}\right)=21.6^{\circ}$. For $\mathrm{C}_{40} \mathrm{H}_{37} \mathrm{NO}_{12} \mathrm{~S}\left(M_{\mathrm{r}}=755.79\right) w_{i} /$ mass \%: calculated: C, 63.57; H, 4.93; S, 4.24, found: C, 63.41; H, 4.90;
$\mathrm{S}, 4.25 .{ }^{1} \mathrm{H}$ NMR $\left(360 \mathrm{MHz},\left(\mathrm{CD}_{3}\right)_{2} \mathrm{SO}\right), \delta: 8.08-7.38$ $\left(\mathrm{m}, 21 \mathrm{H}_{\text {arom }}, \mathrm{NH}\right), 6.03(\mathrm{t}, 1 \mathrm{H}, J=9.4 \mathrm{~Hz}), 5.62(\mathrm{t}$, $1 \mathrm{H}, J=9.3 \mathrm{~Hz}), 5.53(\mathrm{t}, 1 \mathrm{H}, J=9.5 \mathrm{~Hz}), 4.52-4.49$ $(\mathrm{m}, 3 \mathrm{H}), 4.42-4.34(\mathrm{~m}, 2 \mathrm{H}), 3.12(\mathrm{dd}, 1 \mathrm{H}, J=4.1 \mathrm{~Hz}$, $J=13.4 \mathrm{~Hz}), 2.95-2.77(\mathrm{~m}, 3 \mathrm{H}), 2.54(\mathrm{~s}, 1 \mathrm{H}), 1.82(\mathrm{~s}$, $3 \mathrm{H}) .{ }^{13} \mathrm{C} \mathrm{NMR}\left(90 \mathrm{MHz},\left(\mathrm{CD}_{3}\right)_{2} \mathrm{SO}\right), \delta: 172.8,169.0$, 165.3, 165.1, 164.8, 164.7 (6 × CO), 133.7-128.5 (24 × $\mathrm{C}_{\text {arom }}$ ), 76.9, 74.4, 74.4, 71.6, 69.3 (C-2, C-3, C-4, C5, C-6), 62.8 (C-7), 52.8 (태COOH), 34.8, 33.4 (C-1, $\left.\mathrm{CH}_{2}\right), 22.4\left(\mathrm{CH}_{3}\right)$.

\section{Compound IVb}

Compound $I$ (100 mg, $0.169 \mathrm{mmol})$ and captopril $I I I b$ (73 $\mathrm{mg}, 0.338 \mathrm{mmol}$ ) were reacted in tolueneMeOH 1 : 1 vol. (6 mL) according to the general method. The crude product was purified by silica gel chromatography in $\mathrm{CH}_{2} \mathrm{Cl}_{2}-\mathrm{MeOH}\left(\varphi_{\mathrm{r}}=9: 1\right), R_{\mathrm{f}}=$ 0.44 , to give $I V b(123 \mathrm{mg}, 90 \%)$ as a colorless syrup. $[\alpha]_{\mathrm{D}}\left(c=0.02, \mathrm{CHCl}_{3}\right)=-44.3^{\circ}$. For $\mathrm{C}_{44} \mathrm{H}_{43} \mathrm{NO}_{12} \mathrm{~S}$ $\left(M_{\mathrm{r}}=809.88\right) w_{\mathrm{i}} /$ mass \%: calculated: C, 65.25; H, 5.35; S, 3.96, found: C, 65.08; H, 5.33; S, 3.94. ${ }^{1} \mathrm{H}$ $\mathrm{NMR}\left(360 \mathrm{MHz}, \mathrm{CD}_{3} \mathrm{OD}\right), \delta: 8.03-7.75$ (m, 8H $\left.\mathrm{H}_{\text {arom }}\right)$, 7.56-7.18 (m, $\left.12 \mathrm{H}_{\text {arom }}\right), 6.04(\mathrm{t}, 1 \mathrm{H}, J=9.5 \mathrm{~Hz}), 5.72$ $(\mathrm{t}, 1 \mathrm{H}, J=9.7 \mathrm{~Hz}), 5.63(\mathrm{t}, 1 \mathrm{H}, J=9.6 \mathrm{~Hz}), 4.61$ (dd, $1 \mathrm{H}, J=2.9 \mathrm{~Hz}, J=12.3 \mathrm{~Hz}), 4.50(\mathrm{dd}, 1 \mathrm{H}, J=$ $5.0 \mathrm{~Hz}, J=12.2 \mathrm{~Hz}), 4.38-4.33(\mathrm{~m}, 2 \mathrm{H}), 4.24-4.19(\mathrm{~m}$, $1 \mathrm{H}), 3.54-3.51(\mathrm{~m}, 2 \mathrm{H}), 2.94-2.77(\mathrm{~m}, 4 \mathrm{H}), 2.64-2.60$ $(\mathrm{m}, 1 \mathrm{H}), 2.10-2.00(\mathrm{~m}, 1 \mathrm{H}), 1.93-1.78(\mathrm{~m}, 3 \mathrm{H}), 1.06$ $(\mathrm{d}, 3 \mathrm{H}, J=6.4 \mathrm{~Hz}) .{ }^{13} \mathrm{C} \mathrm{NMR}\left(90 \mathrm{MHz}, \mathrm{CD}_{3} \mathrm{OD}\right)$, $\delta$ : 176.1, 175.9, 167.5, 167.2, 166.8, 166.8 (6× CO), 134.7-129.4 (24 $\left.\mathrm{C}_{\text {arom }}\right), 80.1,77.0,76.0,73.3,71.3$ (C2, C-3, C-4, C-5, C-6), 64.6 (C-7), 60.4 (ㅌCOOH), $39.6\left(\mathrm{CHCH}_{3}\right), 48.4,37.7,34.8,30.2,25.6(\mathrm{C}-1,4 \mathrm{x}$ $\left.\mathrm{CH}_{2}\right), 17.3\left(\mathrm{CH}_{3}\right)$.

\section{Compound IVc}

Compound I (100 mg, $0.169 \mathrm{mmol})$ and glutathione IIIC (104 mg, $0.338 \mathrm{mmol})$ were reacted in DMF- $\mathrm{H}_{2} \mathrm{O} 2: 1$ vol. (12 mL) according to the general method. The crude product was purified by silica gel chromatography in $\mathrm{CH}_{2} \mathrm{Cl}_{2}-\mathrm{MeOH}-\mathrm{H}_{2} \mathrm{O}\left(\varphi_{\mathrm{r}}\right.$ $=8: 5: 0.4), R_{\mathrm{f}}=0.43$, to give $I V c(135 \mathrm{mg}, 89 \%)$ as a white solid. $[\alpha]_{\mathrm{D}}(c=0.10, \mathrm{MeOH})=+91.7^{\circ}$. For $\mathrm{C}_{45} \mathrm{H}_{45} \mathrm{~N}_{3} \mathrm{O}_{15} \mathrm{~S}\left(M_{\mathrm{r}}=899.91\right) w_{i} /$ mass \%: calculated: C, 60.06; H, 5.04; S, 3.56, found: C, 59.98; H, 5.02; S, 3.54. ${ }^{1} \mathrm{H} \mathrm{NMR}\left(360 \mathrm{MHz}, \mathrm{CD}_{3} \mathrm{OD}\right), \delta: 7.98-7.37(\mathrm{~m}$, $\left.22 \mathrm{H}_{\text {arom }}, 2 \times \mathrm{NH}\right), 5.98(\mathrm{t}, 1 \mathrm{H}, J=9.1 \mathrm{~Hz}), 5.61(\mathrm{t}$, $1 \mathrm{H}, J=9.2 \mathrm{~Hz}), 5.54(\mathrm{t}, 1 \mathrm{H}, J=9.4 \mathrm{~Hz}), 4.52-4.33(\mathrm{~m}$, $5 \mathrm{H}), 3.72-3.55(\mathrm{~m}, 4 \mathrm{H}), 3.49-3.34(\mathrm{~m}, 2 \mathrm{H}), 3.24-3.10$ $(\mathrm{m}, 1 \mathrm{H}), 3.01-2.89(\mathrm{~m}, 1 \mathrm{H}), 2.84-2.68(\mathrm{~m}, 2 \mathrm{H}), 2.56(\mathrm{~s}$, $2 \mathrm{H}), 2.34-2.22(\mathrm{~m}, 1 \mathrm{H}), 2.14-2.03(\mathrm{~m}, 1 \mathrm{H}), 1.95-1.81$ $(\mathrm{m}, 1 \mathrm{H}) .{ }^{13} \mathrm{C} \mathrm{NMR}\left(90 \mathrm{MHz}, \mathrm{CD}_{3} \mathrm{OD}\right), \delta: 172.6,172.5$, $169.8,165.6,165.3,165.0,164.9(8 \times \mathrm{CO}), 133.8-128.6$ (24 C, arom), 77.1, 74.7, 74.7, 71.7, 69.5 (C-2, C-3, C-

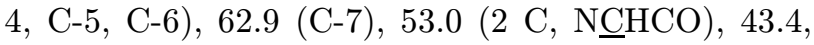
34.4, $29.1\left(\mathrm{C}-1,4 \times \mathrm{CH}_{2}\right)$. 


\section{Compound IVd}

Compound $I(120 \mathrm{mg}, 0.203 \mathrm{mmol})$ and sodium 2sulfanylethanesulfonate IIId (67 mg, $0.406 \mathrm{mmol})$ were reacted in $\mathrm{MeOH}-\mathrm{DMF} 1: 1$ vol. $(6 \mathrm{~mL})$ according to the general method. The crude product was purified by silica gel chromatography in $\mathrm{CH}_{2} \mathrm{Cl}_{2}-\mathrm{MeOH}$ $\left(\varphi_{\mathrm{r}}=85: 15\right), R_{\mathrm{f}}=0.67$, to give $I V d(138 \mathrm{mg}$, $90 \%)$ as a colorless syrup. For $\mathrm{C}_{37} \mathrm{H}_{33} \mathrm{NaO}_{12} \mathrm{~S}_{2}\left(M_{\mathrm{r}}\right.$ $=756.77) w_{i} /$ mass \%: calculated: $\mathrm{C}, 58.72 ; \mathrm{H}, 4.40$; S, 8.47, found: C, 58.61; H, 4.41; S, 8.45. ${ }^{1} \mathrm{H}$ NMR ( $\left.360 \mathrm{MHz},\left(\mathrm{CD}_{3}\right)_{2} \mathrm{SO}\right), \delta: 8.05-7.38\left(\mathrm{~m}, 20 \mathrm{H}_{\text {arom }}\right), 6.06$ $(\mathrm{t}, 1 \mathrm{H}, J=9.4 \mathrm{~Hz}), 5.62(\mathrm{t}, 1 \mathrm{H}, J=9.3 \mathrm{~Hz}), 5.53$ $(\mathrm{t}, 1 \mathrm{H}, J=9.3 \mathrm{~Hz}), 4.55(\mathrm{br} \mathrm{s}, 3 \mathrm{H}), 4.39(\mathrm{~m}, 1 \mathrm{H})$, $2.94-2.76(\mathrm{~m}, 6 \mathrm{H}) .{ }^{13} \mathrm{C} \mathrm{NMR}\left(90 \mathrm{MHz},\left(\mathrm{CD}_{3}\right)_{2} \mathrm{SO}\right)$ : $165.4,165.2,164.9,164.8(4 \times \mathrm{CO}), 133.7-128.5(24$ $\mathrm{C}_{\text {arom }}$ ), 76.7, 74.4, 74.4, 71.9, 69.4 (C-2, C-3, C-4, C-5, C-6), 62.9 (C-7), $51.7\left(\mathrm{NaO}_{3} \mathrm{SCH}_{2}\right), 33.1$ (C-1), 28.1 $\left(\mathrm{CH}_{2} \mathrm{~S}\right)$.

\section{Compound IVe}

Compound $I$ (100 mg, $0.169 \mathrm{mmol})$ and 2,3-di- $O$ acetyl-1-thioglycerol IIIe (65 mg, $0.338 \mathrm{mmol}$ ) were reacted in toluene $(5 \mathrm{~mL})$ according to the general method. The crude product was purified by silica gel chromatography in hexane-acetone $\left(\varphi_{\mathrm{r}}=2: 1\right), R_{\mathrm{f}}=$ 0.50 , to give $I V e(114 \mathrm{mg}, 86 \%)$ as a colorless syrup. $[\alpha]_{\mathrm{D}}\left(c=0.03, \mathrm{CHCl}_{3}\right)=24.5^{\circ}$. For $\mathrm{C}_{42} \mathrm{H}_{40} \mathrm{O}_{13} \mathrm{~S}\left(M_{\mathrm{r}}\right.$ $=784.82) w_{i} /$ mass \%: calculated: $\mathrm{C}, 64.28 ; \mathrm{H}, 5.14$; S, 4.09, found: C, 64.13; H, 5.12; S, 4.08. ${ }^{1} \mathrm{H}$ NMR $\left(360 \mathrm{MHz}, \mathrm{CDCl}_{3}\right), \delta: 8.07-7.80\left(\mathrm{~m}, 8 \mathrm{H}_{\text {arom }}\right), 7.58-$ $7.23\left(\mathrm{~m}, 12 \mathrm{H}_{\text {arom }}\right), 5.92(\mathrm{t}, 1 \mathrm{H}, J=9.5 \mathrm{~Hz}), 5.69(\mathrm{t}$, $1 \mathrm{H}, J=9.7 \mathrm{~Hz}), 5.62-5.55(\mathrm{~m}, 1 \mathrm{H}), 5.21-5.12(\mathrm{~m}, 1 \mathrm{H})$, $4.71-4.66(\mathrm{~m}, 1 \mathrm{H}), 4.48-4.41(\mathrm{~m}, 1 \mathrm{H}), 4.31-4.26(\mathrm{~m}$, $1 \mathrm{H}), 4.20-4.02(\mathrm{~m}, 3 \mathrm{H}), 2.92-2.78(\mathrm{~m}, 4 \mathrm{H}), 2.01,2.00$ $1.99(3 \times \mathrm{s}, 6 \mathrm{H}) \cdot{ }^{13} \mathrm{C} \mathrm{NMR}\left(90 \mathrm{MHz}, \mathrm{CDCl}_{3}\right), \delta: 170.4$, $170.1,166.0,165.8,165.2,165.1$ ( $6 \times \mathrm{CO}), 133.4-128.2$ $\left(24 \mathrm{C}_{\text {arom }}\right), 79.7,79.7,76.1,74.1,71.6,71.5,70.5,70.3$, 69.4 (C-2, C-3, C-4, C-5, C-6, CHOAc), 63.7, 62.9 (C7, $\left.\underline{\mathrm{CH}}_{2} \mathrm{OAc}\right), 33.6,33.5,33.4,33.3\left(\mathrm{C}-1, \mathrm{CH}_{2} \mathrm{~S}\right), 20.8$, $20.6\left(2 \times \mathrm{CH}_{3}\right)$.

\section{Compound IVf}

Compound $I$ (100 mg, $0.169 \mathrm{mmol})$ and 2,2'(ethylenedioxy)diethanethiol IIIf $(14 \mu \mathrm{L}, 0.085 \mathrm{mmol})$ were reacted in toluene-MeOH $1: 1$ vol. $(4 \mathrm{~mL})$ according to the general method, using $3 \times 0.1$ eq. of DPAP $(3 \times 5 \mathrm{mg})$ and irradiation for $3 \times 15 \mathrm{~min}$. The crude product was purified by silica gel chromatography in hexane-acetone $\left(\varphi_{\mathrm{r}}=65: 35\right), R_{\mathrm{f}}=0.20$, to give $\operatorname{IVf}(70 \mathrm{mg}, 61 \%)$ as a colorless syrup. $[\alpha]_{\mathrm{D}}(c$ $\left.=0.20, \mathrm{CHCl}_{3}\right)=+18.8^{\circ}$. For $\mathrm{C}_{76} \mathrm{H}_{70} \mathrm{NO}_{20} \mathrm{~S}_{2}\left(M_{\mathrm{r}}\right.$ $=1367.49) w_{i} /$ mass \%: calculated: $\mathrm{C}, 66.75 ; \mathrm{H}, 5.16$; S, 4.69, found: C, 66.61; H, 5.14; S, 4.67. ${ }^{1} \mathrm{H}$ NMR $\left(360 \mathrm{MHz}, \mathrm{CDCl}_{3}\right), \delta: 8.05-8.03\left(\mathrm{~m}, 4 \mathrm{H}_{\text {arom }}\right)$. 7.94$7.89\left(\mathrm{~m}, 8 \mathrm{H}_{\text {arom }}\right), 7.82-7.79\left(\mathrm{~m}, 4 \mathrm{H}_{\text {arom }}\right), 7.56-7.46$ $\left(\mathrm{m}, 6 \mathrm{H}_{\text {arom }}\right), 7.43-7.31\left(\mathrm{~m}, 14 \mathrm{H}_{\text {arom }}\right), 7.27-7.23(\mathrm{~m}$, $\left.4 \mathrm{H}_{\text {arom }}\right), 5.90(\mathrm{t}, 2 \mathrm{H}, J=9.6 \mathrm{~Hz}), 5.66(\mathrm{t}, 2 \mathrm{H}, J=$ $9.8 \mathrm{~Hz}), 5.54(\mathrm{t}, 2 \mathrm{H}, J=9.6 \mathrm{~Hz}), 4.63(\mathrm{dd}, 2 \mathrm{H}, J=$ $2.9 \mathrm{~Hz}, J=12.2 \mathrm{~Hz}), 4.44(\mathrm{dd}, 2 \mathrm{H}, J=5.3 \mathrm{~Hz}, J=$ $12.2 \mathrm{~Hz}), 4.17-4.12(\mathrm{~m}, 2 \mathrm{H}), 4.03-3.97$ (m, 2H), 3.52 $(\mathrm{t}, 4 \mathrm{H}, J=6.7 \mathrm{~Hz}), 3.40(\mathrm{~s}, 4 \mathrm{H}), 2.87-2.72(\mathrm{~m}, 8 \mathrm{H})$. ${ }^{13} \mathrm{C} \mathrm{NMR}\left(90 \mathrm{MHz}, \mathrm{CDCl}_{3}\right), \delta: 166.0,165.8,165.3$, $165.1(8 \times \mathrm{CO}), 133.4-128.2\left(48 \mathrm{C}_{\text {arom }}\right), 79.7,76.1$, 74.2, 71.8, 69.6 [2 $\times(\mathrm{C}-2, \mathrm{C}-3, \mathrm{C}-4, \mathrm{C}-5, \mathrm{C}-6)], 70.7$, $70.0\left[2 \times\left(\mathrm{CH}_{2} \mathrm{O}, \mathrm{OCH}_{2}\right)\right], 63.2(2 \times \mathrm{C}-7), 33.7,32.6$ $\left[2 \times\left(\mathrm{C}-1, \mathrm{SCH}_{2}\right)\right]$.

\section{Compound IVg}

Compound I (50 mg, $0.09 \mathrm{mmol})$ and 2,3,4,6-tetra$O$-acetyl-1-thiomannopyranose IIIg (44 mg, $0.14 \mathrm{mmol}$ ) were reacted according to the general method. The crude product was purified by silica gel chromatography in hexane-EtOAc $\left(\varphi_{\mathrm{r}}=6: 4\right), R_{\mathrm{f}}=0.28$, to provide compound $I V g(59 \mathrm{mg}, 74 \%)$ as a colorless syrup. $[\alpha]_{\mathrm{D}}\left(c=0.03, \mathrm{CHCl}_{3}\right)=48.7^{\circ}$. For $\mathrm{C}_{49} \mathrm{H}_{48} \mathrm{O}_{18} \mathrm{~S}\left(M_{\mathrm{r}}\right.$ $=1367.49) w_{i} /$ mass $\%$ : calculated $\mathrm{C}, 61.50 ; \mathrm{H}, 5.06$; S, 3.35, found C, 61.65; H, 5.04; S, 3.34. ${ }^{1} \mathrm{H}$ NMR $\left(360 \mathrm{MHz}, \mathrm{CDCl}_{3}\right), \delta: 8.00-8.10\left(\mathrm{~m}, 2 \mathrm{H}_{\text {arom }}\right), 7.85-$ 7.97 (m, $\left.4 \mathrm{H}_{\text {arom }}\right), 7.72-7.84\left(\mathrm{~m}, 2 \mathrm{H}_{\text {arom }}\right), 7.19-7.61$ $\left(\mathrm{m}, 20 \mathrm{H}_{\text {arom }}\right), 5.89(\mathrm{t}, 1 \mathrm{H}, J=9.6 \mathrm{~Hz}), 5.70(\mathrm{t}, 1 \mathrm{H}$, $J=9.8 \mathrm{~Hz}), 5.54(\mathrm{~s}, 1 \mathrm{H}, \mathrm{H}-1), 5.45(\mathrm{t}, 1 \mathrm{H}, J=9.6$ $\mathrm{Hz}), 5.39(\mathrm{dd}, 1 \mathrm{H}, J=3.2, J=1.4 \mathrm{~Hz}), 5.29(\mathrm{t}, 1 \mathrm{H}$, $J=9.8 \mathrm{~Hz}), 5.21(\mathrm{dd}, 1 \mathrm{H}, J=10.0 \mathrm{~Hz}, J=3.3 \mathrm{~Hz})$, $4.70(\mathrm{dd}, 1 \mathrm{H}, J=12.3, J=2.7 \mathrm{~Hz}), 4.20-4.44(\mathrm{~m}$, $3 \mathrm{H}), 3.9-4.18(\mathrm{~m}, 3 \mathrm{H}), 2.96(\mathrm{dd}, 1 \mathrm{H}, J=14.6, J=$ $9.0 \mathrm{~Hz}$ ), $2.81\left(\mathrm{dd},, 1 \mathrm{H}, J=14.6, J=2.5 \mathrm{~Hz}, \mathrm{SCH}_{2}\right.$ ), 1.98, 2.01, 2.05, $2.11\left(4 \times \mathrm{s}, 12 \mathrm{H}, \mathrm{CH}_{3, \mathrm{ac}}\right) \cdot{ }^{13} \mathrm{C} \mathrm{NMR}$ $\left(90 \mathrm{MHz}, \mathrm{CDCl}_{3}\right), \delta: 169.9,169.6,165.8,165.1$ (CO), 133.6, 133.4, 133.1, 129.7, 129.6, 128.8, 128.4, 128.4, $128.2\left(\mathrm{C}_{\text {arom }}\right), 83.1\left(\mathrm{C}-1^{\prime}\right), 79.6,76.3,74.1,72.3,70.1$, $69.2,69.1,66.3,62.7,62.5\left(\mathrm{C}-6, \mathrm{C}-6^{\prime}\right), 31.9\left(\mathrm{CH}_{2} \mathrm{~S}\right)$, $20.7\left(\mathrm{CH}_{3, \text { ac }}\right)$.

\section{Compound Va}

Compound II (256 mg, $1.0 \mathrm{mmol})$ and $N$ - $t$ butoxycarbonyl-L-cysteine ethyl ester IIIh (443 mg, $2.0 \mathrm{mmol}$ ) were reacted according to the general method. The crude product was purified by silica gel chromatography in hexane-EtOAc $\left(\varphi_{\mathrm{r}}=7: 3\right), R_{\mathrm{f}}$ $=0.46$, to provide compound $V a(475 \mathrm{mg}, 94 \%$, ) as a yellow syrup. $[\alpha]_{\mathrm{D}}\left(c=0.50, \mathrm{CHCl}_{3}\right)=52.0^{\circ}$. For $\mathrm{C}_{23} \mathrm{H}_{39} \mathrm{NO}_{9} \mathrm{~S}\left(M_{\mathrm{r}}=505.62\right) w_{i} /$ mass \%: calculated: C, 54.63; H, 7.77; S, 6.34, found C, 54.51; H, 7.76; $\mathrm{S}, 6.32 .{ }^{1} \mathrm{H}$ NMR $\left(400 \mathrm{MHz}, \mathrm{CDCl}_{3}\right), \delta: 7.29(\mathrm{~d}, 1 \mathrm{H}$, $J=3.9 \mathrm{~Hz}, \mathrm{NH}), 5.76(\mathrm{~d}, 1 \mathrm{H}, J=3.6 \mathrm{~Hz}, \mathrm{H}-1), 5.40$ $(\mathrm{d}, 1 \mathrm{H} J=7.6 \mathrm{~Hz}), 4.78(\mathrm{t}, 1 \mathrm{H}, J=4.1 \mathrm{~Hz}), 4.52$ $(\mathrm{m}, 1 \mathrm{H}), 4.22(\mathrm{~m}, 2 \mathrm{H}), 4.10(\mathrm{~m}, 1 \mathrm{H}), 3.99(\mathrm{~m}, 1 \mathrm{H})$, $3.90(\mathrm{~m}, 1 \mathrm{H}), 3.69(\mathrm{~m}, 1 \mathrm{H}), 3.03(\mathrm{~m}, 4 \mathrm{H}), 2.74(\mathrm{t}, J=$ $8.4 \mathrm{~Hz}, 1 \mathrm{H}), 2.09(\mathrm{~m}, 1 \mathrm{H}), 1.52-1.28\left(\mathrm{~m}, 24 \mathrm{H}, \mathrm{CH}_{3}\right)$. ${ }^{13} \mathrm{C}$ NMR (101 MHz, $\left.\mathrm{CDCl}_{3}\right), \delta: 171.0(\mathrm{CO}), 112.0$, $109.6\left(2 \mathrm{C}_{\mathrm{q}}\right), 104.7(\mathrm{C}-1), 81.1,80.8,77.6$ (C-2, C-3 
and $\mathrm{C}-4), 67.5(\mathrm{C}-6), 61.6\left(\mathrm{O}-\mathrm{CH}_{2}\right), 53.39,49.8(\mathrm{C}-3$ and $\mathrm{CH}-\mathrm{NH}), 35.4,28.0\left(2 \times \mathrm{S}-\mathrm{CH}_{2}\right), 28.2,26.7$, 26.6, 26.3, 25.1, $14.1\left(\mathrm{CH}_{3}\right)$.

\section{Compound $\mathrm{Vb}$}

Compound II (256 mg, $1.00 \mathrm{mmol}$ ) and sodium 2-sulfanylethanesulfonate IIId (328 mg, $2.00 \mathrm{mmol})$ were reacted in $\mathrm{MeOH}(8 \mathrm{~mL})$ according to the general method. The crude product was purified by silica gel chromatography in $\mathrm{CH}_{2} \mathrm{Cl}_{2}-\mathrm{MeOH}\left(\varphi_{\mathrm{r}}=8: 2\right), R_{\mathrm{f}}=$ 0.44 , to give $V b(387 \mathrm{mg}, 92 \%)$ as a white solid. $[\alpha]_{\mathrm{D}}$ $\left(c=0.33, \mathrm{CHCl}_{3}\right)=52.4^{\circ}$. For $\mathrm{C}_{15} \mathrm{H}_{25} \mathrm{NaO}_{8} \mathrm{~S}_{2}\left(M_{\mathrm{r}}\right.$ $=420.47) w_{i} /$ mass \%: calculated: C, 42.85; H, 5.99; S, 15.25, found: C, 42.73; H, 5.97; S, 15.21. ${ }^{1} \mathrm{H}$ NMR $\left(360 \mathrm{MHz}, \mathrm{CD}_{3} \mathrm{OD}\right), \delta: 5.76\left(\mathrm{~d}, 1 \mathrm{H}, J_{1,2}=3.3 \mathrm{~Hz}\right.$, $\mathrm{H}-1), 4.81$ (d, 1H, J = 3.7 Hz), 4.10-4.04 (m, 2H), $3.85(\mathrm{dd}, 1 \mathrm{H}, J=8.3 \mathrm{~Hz}, J=10.7 \mathrm{~Hz}), 3.72(\mathrm{dd}, 1 \mathrm{H}$, $J=6.2 \mathrm{~Hz}, J=9.4 \mathrm{~Hz}), 3.12-2.92(\mathrm{~m}, 5 \mathrm{H}), 2.73(\mathrm{t}, 1 \mathrm{H}$, $J=12.1 \mathrm{~Hz}$ ), 2.18-2.13 (m, 1H, H-3), 1.48, 1.39, 1.33, $1.33(4 \times \mathrm{s}, 12 \mathrm{H}) .{ }^{13} \mathrm{C} \mathrm{NMR}\left(90 \mathrm{MHz}, \mathrm{CD}_{3} \mathrm{OD}\right), \delta$ : 113.0, $110.7\left(2 \mathrm{C}_{\mathrm{q}}\right), 106.3(\mathrm{C}-1), 82.5,82.2$ (C-2, C-4), 78.9 (C-5), 68.1 (C-6), $52.8\left(\mathrm{NaO}_{3} \mathrm{SCH}_{2}\right), 50.2$ (C-3), $27.8,27.8\left(\mathrm{SCH}_{2}, \mathrm{CH}_{2} \mathrm{~S}\right), 27.1,26.9,26.6,25.5(4 \times$ $\left.\mathrm{CH}_{3}\right)$.

\section{Compound Vc}

Compound II (128 mg, $0.50 \mathrm{mmol})$ and 2,2'(ethylenedioxy)diethanethiol IIIf $(51 \mu \mathrm{L}, 0.25 \mathrm{mmol}$, 0.5 eq.) were reacted in toluene-MeOH $1: 1$ vol. $(4 \mathrm{~mL})$ according to the general method using $3 \times 0.1$ eq. of $\operatorname{DPAP}(3 \times 5 \mathrm{mg})$ and irradiation for $3 \times 15$ min. The crude product was purified by silica gel chromatography in hexane-acetone $\left(\varphi_{\mathrm{r}}=8: 2\right), R_{\mathrm{f}}$ $=0.30$, to give compound $V c(80 \mathrm{mg}, 46 \%)$ as a colorless syrup. $[\alpha]_{\mathrm{D}}\left(c=0.38, \mathrm{CHCl}_{3}\right)=73.4^{\circ}$. For $\mathrm{C}_{32} \mathrm{H}_{54} \mathrm{O}_{12} \mathrm{~S}_{2}\left(M_{\mathrm{r}}=694.89\right) w_{i} /$ mass \%: calculated: C, 55.31; H, 7.83; S, 9.23, found C, 55.22; H, 7.80; S, 9.15. ${ }^{1} \mathrm{H} \mathrm{NMR}\left(360 \mathrm{MHz}, \mathrm{CDCl}_{3}\right), \delta: 5.76\left(\mathrm{~d}, 2 \mathrm{H}, J_{1,2}\right.$ $=3.6 \mathrm{~Hz}, \mathrm{H}-1), 4.79(\mathrm{~d}, 2 \mathrm{H}, J=3.9 \mathrm{~Hz}), 4.09(\mathrm{dd}, 2 \mathrm{H}$, $J=6.2 \mathrm{~Hz}, J=8.3 \mathrm{~Hz}), 4.03-3.97(\mathrm{~m}, 2 \mathrm{H}), 3.91(\mathrm{dd}$, $2 \mathrm{H}, J=5.2 \mathrm{~Hz}, J=8.3 \mathrm{~Hz}), 3.73-3.64(\mathrm{~m}, 10 \mathrm{H}), 2.99$ $(\mathrm{dd}, 2 \mathrm{H}, J=3.7, J=12.9 \mathrm{~Hz}), 2.81-2.73(\mathrm{~m}, 6 \mathrm{H})$, 2.15-2.07 (m, 2H), 1.51, 1.41, $1.34(3 \times \mathrm{s}, 24 \mathrm{H}) .{ }^{13} \mathrm{C}$ $\mathrm{NMR}\left(90 \mathrm{MHz}, \mathrm{CDCl}_{3}\right), \delta: 111.9,109.5\left(4 \mathrm{C}_{\mathrm{q}}\right), 104.7$ $(2 \times \mathrm{C}-1), 81.1,80.8[2 \times(\mathrm{C}-2, \mathrm{C}-4)], 77.7(2 \times \mathrm{C}-5)$, 70.7, $70.2\left[2 \times\left(\mathrm{CH}_{2} \mathrm{O}, \mathrm{OCH}_{2}\right)\right], 67.5(2 \times \mathrm{C}-6), 49.6$ $(2 \times \mathrm{C}-3), 32.0,27.6\left[2 \times\left(\mathrm{CH}_{2} \mathrm{~S}, \mathrm{SCH}_{2}\right)\right], 26.7,26.6$, $26.4,25.1\left(8 \times \mathrm{CH}_{3}\right)$.

\section{Compound Vd}

Compound II (256 mg, $0.10 \mathrm{mmol}$ ) and 2,3,4,6tetra-O-acetyl-1-thiomannopyranose IIIg $(520 \mathrm{mg}$, $0.15 \mathrm{mmol}$ ) were reacted according to the general method. The crude product was purified by silica gel chromatography in $\mathrm{CH}_{2} \mathrm{Cl}_{2}$-acetone $\left(\varphi_{\mathrm{r}}=98: 2\right)$, to give compound $V d(412 \mathrm{mg}, 95 \%), R_{\mathrm{f}}=0.32$, as a white solid. $[\alpha]_{\mathrm{D}}\left(c=0.34, \mathrm{CHCl}_{3}\right)=114.1^{\circ}$. For $\mathrm{C}_{27} \mathrm{H}_{40} \mathrm{O}_{14} \mathrm{~S}\left(M_{\mathrm{r}}=620.66\right) w_{i} /$ mass \%: calculated: C, $52.25 ; \mathrm{H}, 6.50 ; \mathrm{S}, 5.17$, found C, 52.11; H, 6.48; S, 5.15 . ${ }^{1} \mathrm{H}$ NMR $\left(360 \mathrm{MHz}, \mathrm{CDCl}_{3}\right), \delta: 5.78(\mathrm{dd}, 1 \mathrm{H}, J=12.5$, $J=3.8 \mathrm{~Hz}$ ), $5.23-5.43(\mathrm{~m}, 4 \mathrm{H}), 4.78$ (dd, $1 \mathrm{H}, J=14.8$, $J=10.8 \mathrm{~Hz}), 4.30-4.46(\mathrm{~m}, 2 \mathrm{H}), 4.03-4.16(\mathrm{~m}, 2 \mathrm{H})$, 3.85-4.04 (m, 2H), $3.69(\mathrm{dd}, J=9.5, J=7.6 \mathrm{~Hz}$, $1 \mathrm{H}$ ), 3.06 (dd, $J=12.7, J=3.6 \mathrm{~Hz}, 1 \mathrm{H}, \mathrm{SCH}_{2}$ ), 2.71$2.89\left(\mathrm{~m}, 1 \mathrm{H}, \mathrm{SCH}_{2}\right), 2.00,2.05,2.11,2.18(4 \times \mathrm{s}, 12 \mathrm{H}$, $\left.\mathrm{CH}_{3, \mathrm{ac}}\right), 1.33,1.34,1.39,1.51\left(4 \times \mathrm{s}, 12 \mathrm{H}, \mathrm{CH}_{3, \text { ip }}\right) .{ }^{13} \mathrm{C}$ NMR (91 MHz, $\mathrm{CDCl}_{3}$ ), $\delta: 170.5,169.9,169.7,169.5$ (CO), 112.0, 109.6 ( $\left.\mathrm{C}_{\mathrm{q}}\right), 104.7(\mathrm{C}-1), 81.8\left(\mathrm{C}-1^{\prime}\right), 81.0$, $80.9,77.6,71.0,69.4,68.9,67.7,66.0$ (C-6), 62.1 (C6'), 48.6 (C-3), 26.7, 26.5, 26.4, $25.0\left(\mathrm{CH}_{3, \text { ip }}\right), 25.7$ $\left(\mathrm{SCH}_{2}\right), 20.8,20.6\left(\mathrm{CH}_{3, \mathrm{ac}}\right)$.

\section{Results and discussion}

To produce carbon-sulfur-linked glycoconjugates by the proposed thio-click approach, 3,4,5,7-tetra- $O$ benzoyl-2,6-anhydro-1-deoxy-D-gluco-hept-1-enitol I (Tóth \& Somsák, 2001; Tóth et al., 2003, 2011) and 3-deoxy-1,2:5,6-di- $O$-isopropylidene-3-methylene$\alpha$-D-ribo-hexofuranose $I I$ (Acton et al., 1979) were reacted with functionalized thiols $(I I I a-I I I h)$. The reactions were carried out at ambient temperature with a 2 : 1 thiol : alkene molar ratio and irradiation at $\lambda_{\max }=365 \mathrm{~nm}$ for $15 \mathrm{~min}$ in the presence of the cleavable photoinitiator 2,2-dimethoxy-2phenylacetophenone (DPAP). In case of the bivalent thiol IIIf, an $1: 2$ thiol : ene molar ratio was used. The choice of the solvent was governed by the solubility of the reactants.

As the ligation of sugars to peptides and proteins is a current frontier in glycobiology, reactions of $I$ with amino acids (IIIa and IIIb) and tripeptide IIIc were studied first. Both $\mathrm{N}$-acetyl-L-cysteine (IIIa) and captopril $(I I I b)$, an angiotensin converting enzyme (ACE) inhibitor, reacted readily with the exocyclic double bond of $I$ in toluene-MeOH and gave the corresponding $C$-glycosyl derivatives $I V a$ and $I V b$ in $88 \%$ and $90 \%$ yields, respectively (Table 1 ). The exclusive formation of the $\beta$-glycosides can be explained by the preferred axial attack on the glucopyranosyl radical (Praly, 2000; Dénès et al., 2014). Finding a suitable medium for hydrothiolation of $I$ with glutathione IIIc turned out to be difficult because of their extremely different solubility. Fortunately, in the $2: 1$ vol. ratio of DMF and water, almost complete conversion of the exoglycal was observed, providing the $C$-S-bridged glycopeptide $I V c$ in an $89 \%$ yield. Sodium sulfonatoethyl mercaptane IIId (Mesna), used as a detoxifying adjuvant in cancer chemotherapy, and exoglycal $I$ were reacted in a $1: 1$ vol. mixture of DMF and MeOH to afford the sulfide derivative $I V d$ in good yield. Reaction between $I$ and racemic 2,3-di- $O$-acetyl-1-thioglycerol IIIe (Mugunthan et al., 2011) in toluene resulted in the 
Table 1. Photoinduced addition of thiols to exoglycal $I$

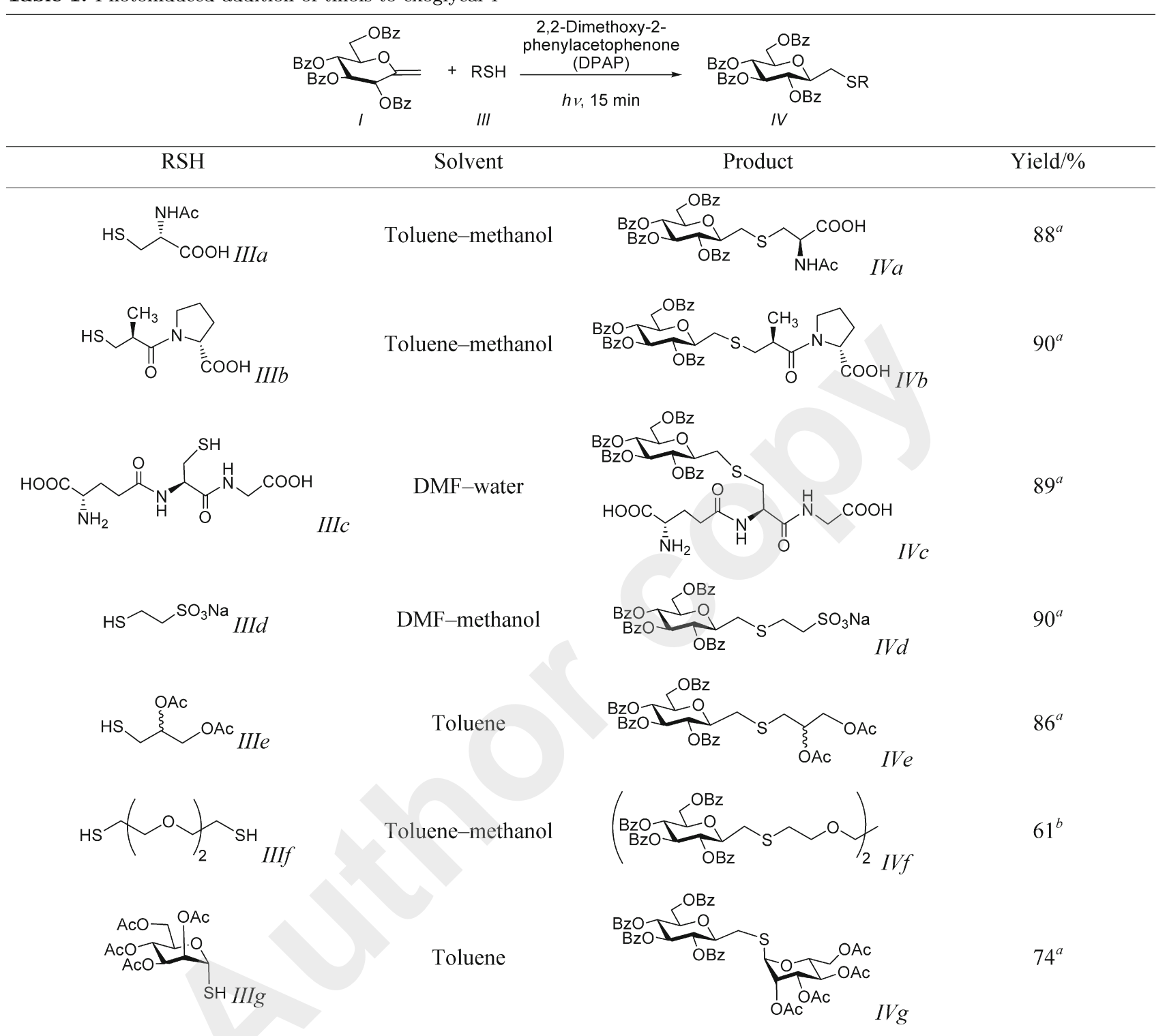

a) Yield of isolated compounds with a $2: 1$ thiol : ene molar ratio using a 0.1 eq. of DPAP; b) $3 \times 15$ min irradiation, 0.5 eq. of thiol, 0.3 eq. of DPAP.

glycerol glycoside derivative $I V e$ as a 1 : 1 diastereoisomeric mixture. To couple two $C$-glycosyl units, compound $I$ was reacted with a 0.5 equivalent of the bivalent thiol IIIf. In this case, the reaction showed low conversion after $15 \mathrm{~min}$; therefore, the DPAP addition and the irradiation were repeated twice more. Thus, after three cycles of irradiation, compound $I V f$ was isolated in a $61 \%$ yield. Finally, the synthesis of a $C-S$ bridged disaccharide was achieved by hydrothiolation of $I$ with 2,3,4,6-tetra- $O$-acetyl-1-thiomannopyranose IIIg (Matta et al., 1975) producing the disaccharide mimetic $I V g$ in a $74 \%$ yield.

Then, the free-radical hydrothiolation reactions of the 3 -exomethylene-derivative $I I$ bearing the exocyclic double bond anchored to a furanose ring were studied. We were pleased to find that an addition of $N$ -
t-butoxycarbonyl-L-cysteine ethyl ester IIIh (Stellenboom et al., 2010) across the double bond of $I I$ resulted into completion within $15 \mathrm{~min}$, providing the D-allo-configured sugar-amino acid conjugate $V a$ exclusively, in a $94 \%$ yield. Stereolectivity of the reaction can be explained by the preferential $\beta$-side hydrogen abstraction from the thiol by the carboncentered radical. Hydrothiolation of $I I$ with both the sulfonic acid salt IIId and the 1-thiomannose $I I I g$ showed high efficacy and full stereoselectivity, affording $V b$ and $V d$ in $92 \%$ and $95 \%$ yields, respectively.

Reaction between $I I$ and dithiol IIIf with a $2: 1$ alkene : thiol mole ratio gave, upon $3 \times 15$ min irradiation, the desired pseudodisaccharide mimetic $V c$ in a $46 \%$ yield. 
Table 2. Photoinduced addition of thiols to 3-exomethylene derivative $I I$

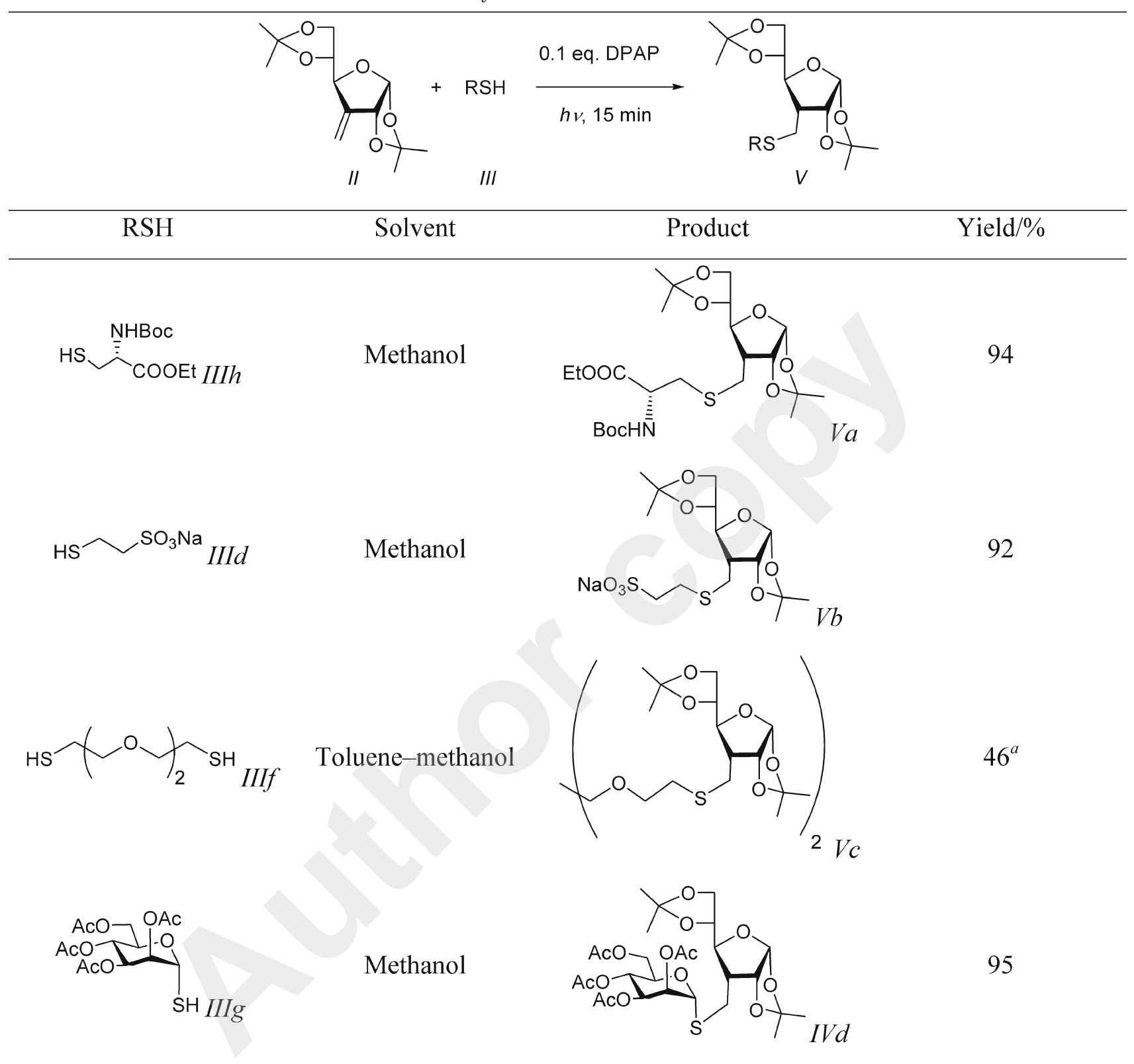

a) $3 \times 15 \mathrm{~min}$ irradiation, 0.5 eq. of thiol, 0.3 eq. of DPAP.

\section{Conclusions}

In conclusion, it has been demonstrated that photoinduced addition of thiols to exocyclic double bonds of furanose or pyranose ring sugars proceeds with total selectivity offering an easy access to carbon-sulfur bridged glycomimetics. Addition of a range of thiols to the benzoyl-subsituted pyranoid-exoglycal $I$ provided exclusively the $\beta$-carbon-sulfur-linked glycoconjugates in high yields. Reactions between thiols and the 3-exomethylene-glucofuranose derivative $I I$ also proceeded with both high efficacy and full stereoselectivity and afforded a series of D-allo-configured 3-deoxy-3- $C$-S-bridged glycoconjugates.
Acknowledgements. This research was supported by the European Union and the State of Hungary, co-financed by the European Social Fund in the framework of TÁMOP 4.2.4. A/211-1-2012-0001 'National Excellence Program'. The project is co-financed by the European Union and the European Social Fund. Financial support of the Hungarian Scientific Research Fund $(K$ 109208, 109450) is also acknowledged. This paper was supported by the János Bolyai Research Scholarship of the Hungarian Academy of Sciences and by the grant TAMOP-4.2.2.A11/1/KONV-2012-0036 provided by the European Union.

\section{References}

Acton, E. M., Goerner, R. N., Uh, H. S., Ryan, K. J., Henry, D. W., Cass, C. E., \& LePage, G. A. (1979). Improved antitumor effects in $3^{\prime}$-branched homologs of $2^{\prime}$-deoxythioguanine. Synthesis and evaluation of thioguanine nucleosides of 2,3- 
dideoxy-3-(hydroxymethyl)-D-erythro-pentofuranose. Journal of Medicinal Chemistry, 22, 518-525. DOI: 10.1021/jm0 $0191 \mathrm{a} 012$

Chakka, N., Johnston, B. D., \& Pinto, B. M. (2005). Synthesis and conformational analysis of disaccharide analogues containing disulfide and selenosulfide functionalities in the interglycosidic linkages. Canadian Journal of Chemistry, 83, 929-936. DOI: 10.1139/v05-107.

Dénès, F., Pichowicz, M., Povie, G., \& Renaud, P. (2014). Thiyl radicals in organic synthesis. Chemical Reviews, 114, 25872693. DOI: $10.1021 / \mathrm{cr} 400441 \mathrm{~m}$.

Dondoni, A., \& Marra, A. (2012). Recent applications of thiolene coupling as a click process for glycoconjugation. Chemical Society Reviews, 41, 573-586. DOI: 10.1039/c1cs15157f.

Ernst, B., \& Magnani, J. L. (2009). From carbohydrate leads to glycomimetic drugs. Nature Reviews Drug Discovery, 8, 661-677. DOI: $10.1038 / \operatorname{nrd} 2852$.

Fiore, M., Marra, A., \& Dondoni, A. (2009). Photoinduced thiol-ene coupling as a click ligation tool for thiodisaccharide synthesis. Journal of Organic Chemistry, 74, 4422-4425. DOI: $10.1021 /$ jo900514w.

Illyés, T. Z., Szabó, T., \& Szilágyi, L. (2011). Glycosylation via mixed disulfide formation using glycosylthiophthalimides and -succinimides as glycosylsulfenyl-transfer reagents. Carbohydrate Research, 346, 1622-1627. DOI: 10.1016/j.carres.2011.04.020.

Lázár, L., Csávás, M., Herczeg, M., Herczegh, P., \& Borbás, A. (2012). Synthesis of $S$-linked glycoconjugates and $S$-disaccharides by thiol-ene coupling reaction of enoses. $\mathrm{Or}$ ganic Letters, 14, 4650-4653. DOI: 10.1021/ol302098u.

Lázár, L., Csávás, M., Hadházi, Á., Herczeg, M., Tóth, M., Somsák, L., Barna, T., Herczegh, P., \& Borbás, A. (2013). Systematic study on free radical hydrothiolation of unsaturated monosaccharide derivatives with exo- and endocyclic double bonds. Organic \& Biomolecular Chemistry, 11, 5339-5350. DOI: $10.1039 / \mathrm{c} 30 \mathrm{~b} 40547 \mathrm{~h}$.

Liu, L., McKee, M., \& Postema, M. H. D. (2001). Synthesis of $C$-saccharides and higher congeners. Current Organic Chemistry, 5, 1133-1167. DOI: 10.2174/1385272013374699.

Lopez, M., Bornaghi, L. F., Driguez, H., \& Poulsen, S. A. (2011). Synthesis of sulfonamide-bridged glycomimetics. The Journal of Organic Chemistry, 76, 2965-2975. DOI: 10.1021/jo2001269.

Matta, K. L., Girotra, R. N., \& Barlow, J. J. (1975). Synthesis of $p$-nitrobenzyl and $p$-nitrophenyl 1-thioglycopyranosides. Carbohydrate Research, 43, 101-109. DOI: 10.1016/s00086215(00)83976-2.

Mugunthan, G., Sriram, D., Yogeeswari, P., \& Kartha, K. P. R. (2011). Synthetic analogues of mycobacterial arabinogalactan linkage-disaccharide part II: Synthesis and preliminary screening of lipophilic $O$-alkyl glycosides. Carbohydrate Research, 346, 2401-2405. DOI: 10.1016/j.carres.2011.08.027.

Praly, J. P. (2000). Structure of anomeric glycosyl radicals and their transformations under reductive conditions. Advances in Carbohydrate Chemistry and Biochemistry, 56, 65-151. DOI: $10.1016 / \mathrm{s} 0065-2318(01) 56003-5$
Prosperi, D., Ronchi, S., Lay, L., Rencurosi, A., \& Russo, G. (2004). Efficient synthesis of unsymmetrical ureidolinked disaccharides. European Journal of Organic Chemistry, 2004, 395-405. DOI: 10.1002/ejoc.200300483.

Robina, I., Vogel, P., \& Witczak, Z. (2001). Synthesis and biological properties of monothiosaccharides. Current Organic Chemistry, 5, 1177-1214. DOI: 10.2174/1385272013374743.

Staderini, S., Chambery, A., Marra, A., \& Dondoni, A. (2012). Free-radical hydrothiolation of glycals: A thiol-ene-based synthesis of $S$-disaccharides. Tetrahedron Letters, 53, 702704. DOI: $10.1016 /$ j.tetlet.2011.11.140.

Stellenboom, N., Hunter, R., \& Caira, M. R. (2010). One-pot synthesis of unsymmetrical disulfides using 1-chlorobenzotriazole as oxidant: Interception of the sulfenyl chloride intermediate. Tetrahedron, 66, 3228-3241. DOI: 10.1016/j.tet.2010. 02.077 .

Szilágyi, L., Illyés, T. Z., \& Herczegh, P. (2001). Elaboration of a novel type of interglycosidic linkage: Syntheses of disulfide disaccharides. Tetrahedron Letters, 42, 3901-3903. DOI: 10.1016/s0040-4039(01)00578-0.

Szilágyi, L., \& Varela, O. (2006). Non-conventional glycosidic linkages: Syntheses and structures of thiooligosaccharides and carbohydrates with three-bond glycosidic connections. Current Organic Chemistry, 10, 1745-1770. DOI: $10.2174 / 138527206778249874$.

Tóth, M., \& Somsák, L. (2001). exo-Glycals from glycosyl cyanides. First generation of $C$-glycosylmethylene carbenes from 2,5- and 2,6-anhydroaldose tosylhydrazones. Journal of the Chemical Society, Perkin Transactions 1, 942-943. DOI: $10.1039 / \mathrm{b} 102963 \mathrm{k}$.

Tóth, M., Kövér, K. E., Bényei, A., \& Somsák, L. (2003). CGlycosylmethylene carbenes: Synthesis of anhydro-aldose tosylhydrazones as precursors; generation and a new synthetic route to exo-glycals. Organic \& Biomolecular Chemistry, 1, 4039-4046. DOI: 10.1039/b307378e.

Tóth, M., Kun, S., Somsák, L., \& Goyard, D. (2011). Preparation of exo-glycals from 2,6-anhydro-aldose-tosylhydrazones. In P. Kovac (Ed.) Carbohydrate chemistry: Proven synthetic methods (pp. 365-373). Boca Raton, FL, USA: CRC Press.

Witczak, Z. J., \& Culhane, J. M. (2005). Thiosugars: New perspectives regarding availability and potential biochemical and medicinal applications. Applied Microbiology and Biotechnology, 69, 237-244. DOI: 10.1007/s00253-005-0156-

Witczak, Z. J., Lorchak, D., \& Nguyen, N. (2007). A click chemistry approach to glycomimetics: Michael addition of $2,3,4,6$-tetra- $O$-acetyl- 1 -thio- $\beta$-D-glucopyranose to 4 -deoxy1,2-O-isopropylidene-L-glycero-pent-4-enopyranos-3-ulose a convenient route to novel 4 -deoxy- $(1 \rightarrow 5)-5$ - $C$-thiodisaccharides. Carbohydrate Research, 342, 1929-1931. DOI: 10.1016/j.carres.2007.06.005.

Witczak, Z. J., \& Bielski, R. (2013). Click chemistry in glycoscience: New developments and strategies. New York, NY, USA: Wiley. 